\title{
Adaptation, Update and Validation of the General Nutrition Questionnaire in a Portuguese Adolescent Sample
}

\section{Vera Ferro-Lebres, Pedro Moreira \& José Carlos Ribeiro}

To cite this article: Vera Ferro-Lebres, Pedro Moreira \& José Carlos Ribeiro (2014) Adaptation, Update and Validation of the General Nutrition Questionnaire in a Portuguese Adolescent Sample, Ecology of Food and Nutrition, 53:5, 528-542, DOI: 10.1080/03670244.2013.873424

To link to this article: https://doi.org/10.1080/03670244.2013.873424

Published online: 08 Aug 2014.

Submit your article to this journal $\llbracket$

Џ Article views: 254

Q View related articles $\square$

View Crossmark data $\longleftarrow$

Citing articles: 2 View citing articles $\longleftarrow$ 


\title{
Adaptation, Update and Validation of the General Nutrition Questionnaire in a Portuguese Adolescent Sample
}

\author{
VERA FERRO-LEBRES \\ CIAFEL - Research Center in Physical Activity, Health and Leisure, Faculty of Sports, \\ University of Porto, and Diagnostic and Therapeutic Technologies Department, School of \\ Health Sciences, Polytechnic Institute of Braganza, Portugal
}

\section{PEDRO MOREIRA}

CIAFEL - Research Center in Physical Activity, Health and Leisure, Department of Sports, and

Department of Nutrition and Food Sciences, University of Porto, Porto, Portugal

\author{
JOSÉ CARLOS RIBEIRO \\ CIAFEL - Research Center in Physical Activity, Health and Leisure, Faculty of Sports, \\ University of Porto, Porto, Portugal
}

This article describes the adaptation of the adult Portuguese version of the General Nutrition Knowledge Questionnaire (GNKQ) for adolescents, and its validation. Respondents were 1,315 adolescents, who completed the questionnaire in two phases. A subsample of 73 adolescents was used to measure test-retest reliability. Concurrent validity was tested using a sample of 32 dietetic students. The adapted version showed high internal consistency (Cronbach's alpha $=0.92)$, test-retest reliability $(R=0.71)$ and concurrent validity ( $U=22766.0 ; p<.01)$. Adolescents' nutrition knowledge can now be assessed with a valid and reliable instrument. Future validation works of this or others questionnaires for children and elderly are warranted.

KEYWORDS adolescents, nutrition knowledge, questionnaire, validation

Address correspondence to Vera Ferro-Lebres, RD, CIAFEL - Research Center in Physical Activity, Health and Leisure, Rua Dr. Plácido Costa, 91, 4200-450 Porto, Portugal. E-mail: vferrolebres@ipb.pt 
Several eating-behavior determinants have been studied, including nutrition knowledge (Raine 2005; Taylor, Evers, and McKenna 2005; Viswanath and Bond 2007). The influence of nutrition knowledge on food behavior has no consensus (Taylor et al. 2005): Some studies found a weak association or no correlation at all (Mullaney, Corish, and Loxley 2009; Pirouznia 2001; SerraMajem et al. 2007); others found a significant positive association (De Vriendt et al. 2009; Shah et al. 2010; Sharma, Gernand, and Day 2008), or a positive association only with fruits and vegetables (De Vriendt et al. 2009; Parmer et al. 2009; Wardle, Parmenter, and Waller 2000) or fat consumption (Wardle et al. 2000).

Reasons have been pointed out for the weak associations: (1) poor nutrition-knowledge conceptualization; (2) lack of nutrition-knowledge relevance for the studied population; (3) poor correspondence between knowledge dimensions and food-consumption domains; (4) a small sample size; (5) data analysis inaccuracies; and (6) questionnaire inadequacy (using non-validated questionnaires) (Wardle et al. 2000; Worsley 2002). Thus, it seems important to validate questionnaires, adapted to sample characteristics such as age and cultural context.

Considering that several recommendations have been made regarding the importance of planning interventions on nutrition education for children and adolescents, namely focusing nutrition knowledge (Pratt, Stevens, and Daniels 2008; WHO 2009), it seems important to validate questionnaires that allow researchers to evaluate the impact of such interventions, allowing researchers to score nutrition knowledge also in younger samples.

The General Nutritional Knowledge Questionnaire (GNKQ; Parmenter and Wardle 1999) is one of the few that tests general knowledge and not a nutrition-knowledge specific area. It includes different sections: (1) dietary recommendations, (2) nutrient content of different food items, (3) dietary best choices, and (4) health/disease issues regarding diet. GNKQ has been proven to be valid and reliable in a UK adult sample (Parmenter and Wardle 1999), in an Australian community sample (Hendrie, Cox, and Coveney 2008), in a Turkish adult Sample (Alsaffar 2012), and in a Portuguese adult sample (Almeida-de-Souza 2009). However, in Portuguese adolescents, to the best of our knowledge, no validation has been published so far for this or other general nutrition-knowledge questionnaire, and few exist for other countries.

Since GNKQ development and validation, scientific knowledge and food practices have been in constant evolution, making necessary the instrument's update according to the latest scientific evidences.

The present work aims to update the Portuguese version of the GNKQ and to determine its validity and reliability in a Portuguese adolescent sample. 


\section{METHODOLOGY}

The process of adaptation, updating and validation was done in two phases. Phase 1 consisted of making minor adjustments, and adapting the language of the original version of the GNKQ questionnaire. This adapted version was pilot-tested with 603 individuals to ensure age-appropriateness. In Phase 2, some food items were included and the score of each item was revised according to the latest scientific evidence (Anderson et al. 2009; Brown et al. 2009; de Sa and Lock 2008; He and MacGregor 2009; Hoffmann et al. 2003; Kipping, Jago, and Lawlor 2008; Kushi et al. 2006; Mirmiran et al. 2009; Ruxton, Gardner, and McNulty 2010). Results of the 603 Phase 1 questionnaires were analyzed regarding item difficulty, and the questionnaire was changed in accordance. The Phase 2 questionnaire was then tested with a sample of 712 individuals.

Sample characterization data for age, sex, and grade was collected in both moments.

\section{Participants}

The sample size was clearly over the minimum 400 individuals recommended for Internal Reliability studies (Charter 2003). The minimum subsample size of 30 for test-retest reliability was also assumed (Charter 2008).

For Phase 1, 603 high school students aged 11 to 19 years (mean $=16.4 \mathrm{SD}=1.71$ ), who attended three different schools in the north of Portugal, comprised the study sample.

For Phase 2, the study sample consisted of 712 high school students aged 10 to 19 years $($ mean $=15.0, S D=2.00$ ), from 12 different schools distributed geographically in the north and center of Portugal. In Phase 2 we aimed to have a broader age-range representation, including more students from the younger-age group, which was considered insufficient in Phase 1.

Table 1 summarizes the Phase 1 and Phase 2 sample characteristics.

\section{Ethical Approval}

This study was conducted according to the guidelines from the Declaration of Helsinki and all procedures were approved by the Research Center in Physical Activity, Health and Leisure Scientific Committee. Written informed consent was obtained from all parents.

Directors of the involved schools gave their ethical approval. Adolescents were given an opportunity to refuse participation.

There was a guarantee of anonymous and confidential data analysis, for both the paper and online versions of the questionnaire. 


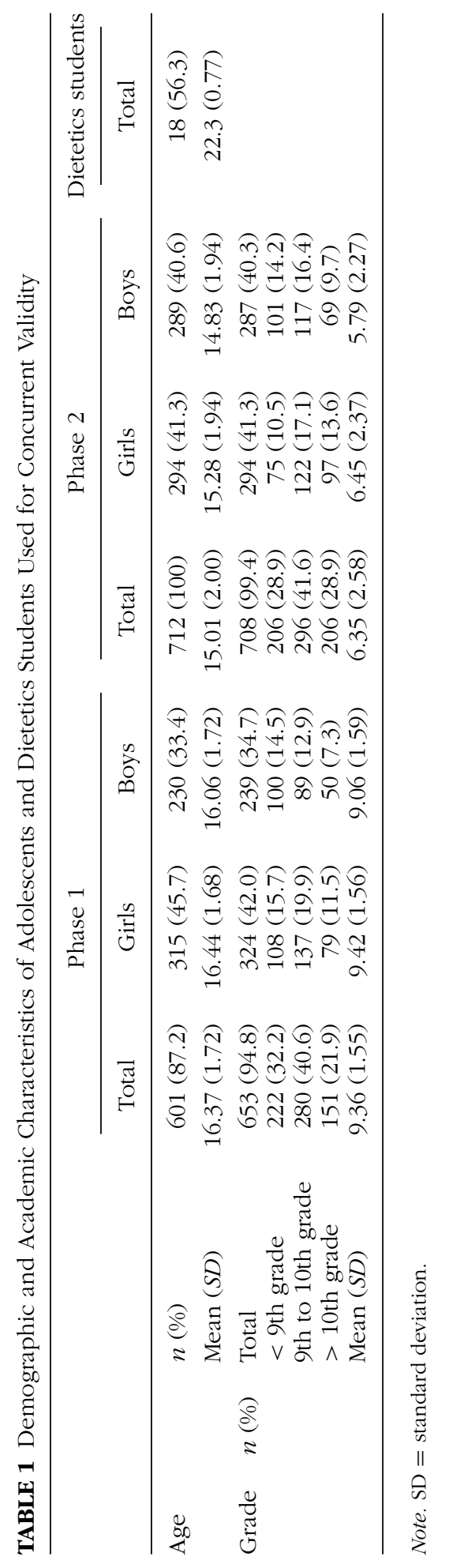




\section{Data Collection and Analysis}

Data was collected between 2009 and 2011. This time frame did not affect the results of this study.

Two versions of the questionnaire were created: a paper version (machine-readable) and an online version. The latter was developed in order to reduce the item-non-response rate, as previously suggested (Denscombe 2009).

The first author and/or the responsible teacher from each class supervised the self-administration of the questionnaire to small groups, in a classroom environment.

In Phase 1 of the study, each answer was coded numerically and converted into two scores: the original score (according to the original version; Parmenter and Wardle 1999) and the Portuguese adapted score (considering the Portuguese Healthy Eating Recommendations; Rodrigues et al. 2006).

In Phase 2, each answer was coded numerically and converted into one score (the Portuguese adapted and updated score), considering the Healthy Eating Portuguese Recommendations (Rodrigues et al. 2006) and the most recent scientific evidence (Anderson et al. 2009; Brown et al. 2009; de Sa and Lock 2008; He and MacGregor 2009; Hoffmann et al. 2003; Kipping et al. 2008; Kushi et al. 2006; Mirmiran et al. 2009; Ruxton, Gardner, and McNulty 2010).

In both phases, each correct item was scored 1 point. Incorrect or missing answers were scored 0 points. Data analysis was performed using IBM Statistical Package for Social Sciences, version 19. Statistical significance was set at $p<.05$.

\section{Content Validity and Questionnaire Refinement}

The content validity is defined as the extent to which the questionnaire covers all dimensions present in the concept it is intended to reflect (Raykov and Marcoulides 2011; Terwee et al. 2007; Thorndike 1995).

The GNKQ intends to measure nutritional knowledge in a broad range of the concept. In this adaptation and validation process, for the Portuguese adolescent population, it was decided to keep the original authors' four areas: (1) expert dietary recommendations; (2) nutrient content of food; (3) healthier food choices; and (4) diet-disease relation. The same was done in the Portuguese adult version (Almeida-de-Souza 2009).

\section{PHASE 1}

For the adaptation and validation for the Portuguese adolescent population, the adult Portuguese version was used and minor adjustments were made. The translation, cross-cultural adaptation, and validation to the adult population processes are described elsewhere (Almeida-de-Souza 2009). 
Adjustments were intended to guarantee the GNKQ was language appropriate to the age range of the sample, and to ensure that the instrument was coherent with the new food guide for the Portuguese population (Rodrigues et al. 2006). All adjustments were done by an expert panel consisting of dietitians/nutritionists and teachers/professors with pedagogical background.

In section 1, the question regarding fruit and vegetable consumption was scored according to the Parmenter and Wardle original score (1999) (i.e., 5 or 6 portions a day) and according to the Portuguese recommendations (Rodrigues et al. 2006) (i.e., from 6 to 10 portions a day); the question regarding the dairy-fat-content recommendation also had two different scores: the original one (i.e., lower fat) and in agreement with the Portuguese recommendations (i.e., half-skimmed).

In section 2 , in the question about the fat content of different food items, the cottage cheese was replaced with a low-fat Portuguese fresh cheese, considering adolescents' limited access to cottage cheese and/or knowledge of its existence, and the significantly higher availability of the low-fat Portuguese fresh cheese.

In section 3 , the question asking for a low-fat, high-fiber snack choice was scored as the original score, considering the muesli bar as the correct answer because experts considered that Portuguese food habits do not include eating a recommended portion of raisins as a snack; as such, choosing carefully between the available options, the muesli bar seemed the most adequate option.

In section 4 , in the question about health problems or diseases related to fruit and vegetable consumption, besides all the possible correct answers considered by the original score, "avitaminosis," or vitamin deficits, was also considered correct. In the question regarding health problems or diseases related to fat consumption "high cholesterol" was also considered a correct answer.

\section{PHASE 2}

In Phase 2, the final version of the General Nutrition Knowledge Questionnaire for Adolescents (GNKQA) was developed; some food items were included by the expert panel, in order to update the questionnaire and to guarantee that all the Portuguese healthy-eating recommendations were covered. In doing so, we expected Portuguese adolescents to more highly identify with the questionnaire. The scoring of some items was also changed in this Phase 2 in order to guarantee the update of the questionnaire to current scientific knowledge.

In section 1 , in the question about healthy-eating recommendations, two items were added: beans and vegetable soup.

In section 2, French fries and a ham-and-cheese puff pastry snack, both widely consumed by Portuguese adolescents, were added to the questions about food items considered to be high in salt and fat. 
In section 4, in the question regarding cancer-prevention behavior, the word "fiber" was replaced by "whole grain cereals," as mentioned in the American Cancer Society Guidelines (Kushi et al. 2006). We also added a "yes-or-no" question about eating behaviors that can contribute to obesity.

\section{Item Difficulty}

An item is not considered useful if it is answered correctly by more than $90 \%$ or less than 10\% of individuals (Domino and Domino 2006).

After collecting Phase 1 data, items that did not meet these criteria were excluded, except if they were considered essential for content validity by the expert panel.

In section 1, the first question asking about experts recommendations on "starchy food" consumption, the panel considered it more appropriate to change the broader expression "Starchy Food" for "whole wheat bread," since adolescents may not knew the meaning of "starchy".

In section 2, all items met the difficulty criteria, therefore no changes were made.

Section 3 had a question on the best food choice for a low-salt diet, and this question was excluded, considering that in section 2 the salt content of several food items was addressed.

In section 4, the open-answer questions on health problems related to fruit and vegetable consumption; low-dietary fiber consumption; high sugar consumption and high fat ingestion were statistically too difficult for this age range. The expert panel considered it more appropriate to convert the open answer into a "yes-or-no" answer. To guarantee coherence in the format, the question on health problems related to salt consumption was also changed. The latest published reviews in each subject were taken into account in the definition of the health problems/diseases mentioned in each question (Anderson et al. 2009; Hoffman et al. 2003; Polonia and Martins 2009; Ruxton et al 2010).

The last question of this section regarding antioxidants did not meet the difficulty criteria, and was considered by the expert panel that it was not age appropriate and eventually was excluded.

For Phase 2 results, all items met the difficulty criteria (Domino and Domino 2006).

\section{Internal Reliability}

Internal reliability of survey instruments evaluates reliability of different items intended to measure the same concept. Cronbach's Alpha is therefore widely accepted as a good statistical method to calculate this reliability (Raykov and Marcoulides 2011; Terwee et al. 2007; Thorndike 1995). 
PHASE 1

The GNKQ has four different sections, each focusing on a different category of nutritional knowledge. Thus the Cronbach's Alpha was performed individually for each section and for the whole GNKQ, according to the original score. For sections 1, 3, and 4, and for the whole GNKQ, Cronbach's alpha was also calculated for the Portuguese updated score. Section 2 did not have any specific Portuguese score.

\section{PHASE 2}

The original four sections were maintained in GNKQA. Cronbach's alpha was calculated separately for each section and for the total GNKQA, using the adapted and updated score.

\section{Test-Retest Reliability}

During Phase 2 a subsample of 73 students (mean age $=16.3 ; S D=$ 2.28) completed the questionnaire on a second occasion between one and two weeks apart, in order to determine reproducibility (Terwee et al. 2007; Thorndike 1995; Raykov and Marcoulides 2011).

After performing normality tests, a Spearman Ró correlation coefficient between scores (total score and score for sections 1, 2, 3, and 4) on both occasions was performed to verify consistency (Raykov and Marcoulides 2011; Terwee et al. 2007; Thorndike 1995).

\section{Concurrent Validity}

In order to verify if the questionnaire actually measures nutrition knowledge, concurrent validity was tested (i.e., the ability of a questionnaire to distinguish between groups, which theoretically would happen) (Raykov and Marcoulides 2011; Terwee et al. 2007; Thorndike 1995).

The GNKQA was applied to a sub sample of 32 Dietetics students from the third and fourth year of the degree, considering these should have significantly higher knowledge. Table 1 shows the characteristics of this subsample.

To assess differences between groups in GNKQA scores, the MannWhitney U test was performed, after testing for normality. It was expected that dietetics students had significantly higher scores than the adolescent's sample.

\section{Content Validity}

\section{RESULTS}

After all the adaptation process, the resulting GNKQA had 137 items, distributed through the four original sections: Section 1, Dietary 
recommendations; section 2, Sources of nutrients; section 3, Choosing everyday foods; and section 4, Diet-disease relationship.

\section{INTERNAL RELIABILITY}

Cronbach's alpha was performed to evaluate internal reliability during Phase 1 and Phase 2. Test value ranged from 0.22 and 0.87 in Phase 1 , and from 0.33 to 0.92 in Phase 2 . Internal reliability was very high in relation with the GNKQA total score, and high in reference to sections 2 and 4 (table 2).

\section{Test-Retest Reliability}

Spearman Ró correlation revealed acceptable test-retest reliability for the questionnaire total score $(R=0.71, p<.001)$ (table 2$)$.

\section{Concurrent Validity}

Dietetics students' scores for each section, and for the total GNKQA, were significantly higher $(p<.01)$ than the ones from the adolescent sample (table 3).

\section{Data Collection Methods}

After testing for normality, Mann-Whitney U test was performed to test the differences between scores of the questionnaires filled in paper version and

TABLE 2 Internal Reliability and Test-Retest Reliability

\begin{tabular}{|c|c|c|c|c|c|}
\hline & & & Phase 1 & Phase 2 & \\
\hline & Score & ( $N$ of items/MS) & Cronbach's $\alpha$ & Cronbach's $\alpha$ & $R$ \\
\hline \multirow[t]{3}{*}{ Section 1} & Original score & $(11 / 11)$ & 0.55 & - & - \\
\hline & Adapted score & $(11 / 11)$ & 0.55 & - & - \\
\hline & Final score & $(13 / 13)$ & - & $0.63^{* *}$ & $0.44^{* * *}$ \\
\hline \multirow[t]{2}{*}{ Section 2} & Original score & $(69 / 69)$ & 0.85 & - & - \\
\hline & Final score & $(73 / 73)$ & - & 0.85 & $0.60^{* * *}$ \\
\hline \multirow[t]{3}{*}{ Section 3} & Original score & $(10 / 10)$ & 0.22 & - & - \\
\hline & Adapted score & $(10 / 10)$ & 0.29 & - & - \\
\hline & Final score & $(9 / 9)$ & - & 0.33 & $0.43^{* * *}$ \\
\hline \multirow[t]{3}{*}{ Section 4} & Original score & $(17 / 20)^{*}$ & 0.71 & - & - \\
\hline & Adapted score & $(17 / 22)^{*}$ & 0.72 & - & - \\
\hline & Final score & $(42 / 42)$ & - & 0.88 & $0.65^{* * *}$ \\
\hline \multirow[t]{3}{*}{ Total } & Original score & $(106 / 110)^{*}$ & 0.87 & - & - \\
\hline & Adapted score & $(106 / 112)^{*}$ & 0.87 & - & - \\
\hline & Final score & $(137 / 137)$ & - & 0.92 & $0.71^{* * *}$ \\
\hline
\end{tabular}

Note. MS = Maximum score.

*One item had zero variance and was removed from the internal reliability analysis.

${ }^{* *}$ Could be acceptable according to some authors; previous validation considered lowest values as moderate reliability.

${ }^{* * *} p<.001$. 


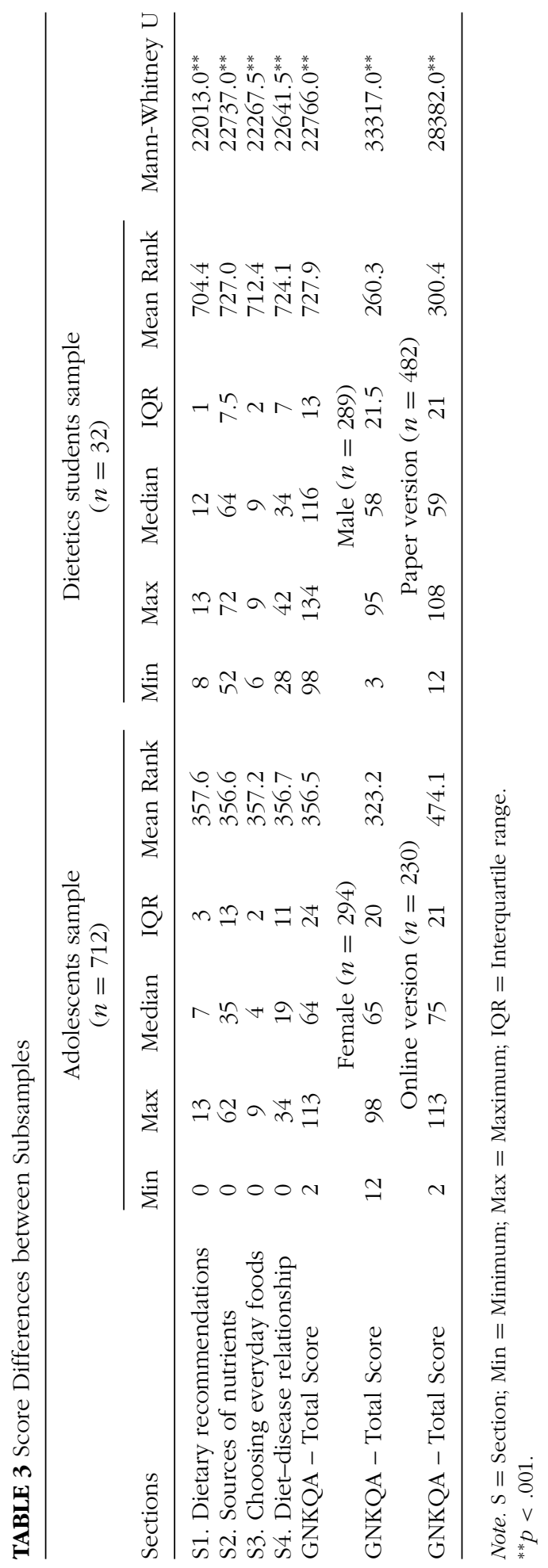


the online version. The online version had significantly higher scores than did the paper version $(p<.00)$. Demographic samples were not significantly different (table 3).

\section{Nutrition Knowledge}

After testing for normality, the Mann-Whitney U test was performed to test the differences between questionnaire scores of adolescent boys and girls. Girls had significantly higher scores $(p<.00)$ (table 3).

\section{DISCUSSION}

The present work adapts, updates, and validates the GNKQ to be used with Portuguese adolescents. As far as we know, there is no published work studying nutritional knowledge in Portuguese adolescents, and we believe that this is due to the inexistence of validated questionnaires. Several previous published works aimed to study the relation between health knowledge and practices, particularly nutrition related studies (De Vriendt et al. 2009; Mullaney, et al. 2009; Parmer et al. 2009; Pirouznia 2001; Raine 2005; SerraMajem et al. 2007; Shah et al. 2010; Sharma et al 2008; Taylor, et al 2005; Viswanath and Bond 2007; Wardle et al 2000). Some critics have been made to the use of non-validated and not age specific questionnaires or to the lack of coverage of all aspects regarding nutrition knowledge (Wardle et al 2000).

The original GNKQ was developed in 1999. Since then several scientific reviews on the aspects covered in the questionnaire were published. During content validity our expert panel considered it important to change some items' scoring and to include some new items; originating a different score for the GNKQA, but keeping the original structure. As researchers, we strongly recommend that before the use of any knowledge questionnaire, an update revision to the scoring should be done.

GNKQA proved to be reliable to use as a whole, to administer sections 2 and 4 individually, if intended to evaluate only the covered nutrition aspects mentioned there.

The two sections with lower reliability were the ones with fewer items, which could in part explain the results. It is described that the test value tends to increase as the number of items increases (Thorndike 1995). In coherence, the previously validated versions of the GNKQ had lower Cronbach's alphas in these sections, comparing with sections 2 and 4 and with the total score (Hendrie et al 2008). Also, in the present work, the scores with the bigger (total GNKQA) and smaller (section 3) number of items, had the highest and lower test values, respectively.

The section 1 reliability coefficient of 0.63 could be acceptable, according to some authors; and previous validation of GNKQ considered the lower 
values of Cronbach's alpha as moderate reliability indicators (Hendrie et al. 2008).

Test-retest reliability was acceptable, showing the ability of GNKQA to consistently assess knowledge over time, enhancing its aptitude to adequately evaluate changes after nutrition-education interventions.

The correlation coefficient of the Portuguese version was slightly inferior to the ones mentioned in the previous validation studies (Alsaffar 2012; Hendrie et al 2008; Parmenter and Wardle 1999), which may be explained by the age differences of the studied samples.

Concurrent validity proved that this questionnaire was able to effectively distinguish between groups with different nutrition-knowledge levels, as its previous versions did (Hendrie et al 2008; Parmenter and Wardle 1999).

Regarding the two data-collection methods, the online version had a significantly higher score than that of the paper version. Previous studies found the same results, and suggested that this finding could be related to a smaller item-non-response rate (Denscombe 2009; Kongsved et al. 2007).

As previously suggested for adults (Parmenter, Waller, and Wardle 2000), as in our adolescent sample, girls had a significantly higher total score. These results suggest that the girls' greater interest in nutrition starts early in adolescence.

We can state as a limitation to the present validation process the fact that the sample of Dietetics students included only girls, but the highereducation institution that collaborated with this research had only two boys in the selected academic years, and they refused to participate.

The length of the questionnaire was also mentioned by the expert panel and by adolescents as a negative factor. On average, it took 20 minutes for an adolescent to answer the whole questionnaire. We should highlight that comments reporting test duration as a constraint were written by the subsample that answered the paper version, and not by those who responded online. The possibility of administering sections 2 and 4 independently can be part of a solution, whenever it would be sufficient to assess only some aspects of nutrition knowledge.

\section{CONCLUSION}

In conclusion, Portuguese adolescents' nutrition knowledge can now be assessed with a valid and reliable instrument. The GNKQA may be of general use for researchers or dietetics and nutrition professionals working within nutrition-education interventions for adolescents; offering the possibility to evaluate the results of interventions in a reliable and consistent way.

We consider that the GNKQA can also be used in clinical contexts, and by non-experts in the nutritional sciences, as the instrument is easy to use and score-the online version, in particular. It has been suggested that 
online assessment is a good methodology, as it saves resources, provides more complete answers, and facilitates data collection in follow-up studies (Kongsved et al. 2007).

The process of translating and validating this adapted version for other languages/countries would be of great benefit to the research of adolescents' diet correlates, particularly in regards to the relationship between nutrition knowledge and diet.

\section{FUNDING}

This work was supported by FCT- Portuguese Foundation for Science and Technology for the PROTEC project grant SFRH/PROTEC/49529/2009, conceded to the first author and project grant FCOMP-01-0124-FEDER-028619 (Ref. FCT: PTDC/DTP-DES/1328/2012).

\section{REFERENCES}

Almeida-de-Souza, J. 2009. Nutritional knowledge: Reproduction and validation questionnaire. [In Portuguese.] Porto, Portugal: Faculty of Medicine, University of Porto.

Alsaffar, A. A. 2012. Validation of a general nutrition knowledge questionnaire in a Turkish student sample. Public Health Nutrition FirstView:1-12.

Anderson, J. W., P. Baird, R. H. Davis, Jr., S. Ferreri, M. Knudtson, A. Koraym, V. Waters, and C. L. Williams. 2009. Health benefits of dietary fiber. Nutrition Reviews 67 (4): 188-205.

Brown, I. J., I. Tzoulaki, V. Candeias, and P. Elliott. 2009. Salt intakes around the world: Implications for public health. International Journal of Epidemiology 38 (3): 791-813.

Charter, R. A. 2003. Study samples are too small to produce sufficiently precise reliability coefficients. The Journal of General Psychology 130 (2): 117.

Charter, R. A. 2008. Statistical approaches to achieving sufficiently high test score reliabilities for research purposes. The Journal of General Psychology 135 (3): 241-251.

de Sa, J., and K. Lock. 2008. Will European agricultural policy for school fruit and vegetables improve public health? A review of school fruit and vegetable programs. The European Journal of Public Health 18 (6): 558-568.

De Vriendt, T., C. Matthys, W. Verbeke, I. Pynaert, and S. De Henauw. 2009. Determinants of nutrition knowledge in young and middle-aged Belgian women and the association with their dietary behaviour. Appetite 52 (3): 788-792.

Denscombe, M. 2009. Item non-response rates: A comparison of online and paper questionnaires. International Journal of Social Research Methodology 12 (4): 281-291.

Domino, G., and M. L. Domino. 2006. Psychological testing: An introduction. New York: Cambridge University Press. 
He, F. J., and G. A. MacGregor. 2009. A comprehensive review on salt and health and current experience of worldwide salt reduction programs. Journal of Human Hypertension 23 (6): 363-384.

Hendrie, G. A., D. N. Cox, and J. Coveney. 2008. Validation of the General Nutrition Knowledge Questionnaire in an Australian community sample. Nutrition $\mathcal{E}$ Dietetics 65 (1): 72-77.

Hoffmann, K., H. Boeing, J. Volatier, and W. Becker. 2003. Evaluating the potential health gain of the World Health Organization's recommendation concerning vegetable and fruit consumption. Public Health Nutrition 6 (8): 765-772.

Kipping, R. R. R. Jago, and D. A. Lawlor. 2008. Obesity in children. Part 2: Prevention and management. BMJ 337.

Kongsved, S. M., M. Basnov, K. Holm-Christensen, and N. H. Hjollund. 2007. Response rate and completeness of questionnaires: A randomized study of Internet versus paper-and-pencil versions. Journal of Medical Internet Research 9 (3): e25.

Kushi, L. H., T. Byers, C. Doyle, E. V. Bandera, M. McCullough, A. McTiernan, T. Gansler, K. S. Andrews, and M. J. Thun. 2006. American Cancer Society Guidelines on nutrition and physical activity for cancer prevention: Reducing the risk of cancer with healthy food choices and physical activity. CA: A Cancer Journal for Clinicians 56 (5): 254-281; quiz 313-314.

Mirmiran, P., N. Noori, M. Beheshti Zavareh, and F. Azizi. 2009. Fruit and vegetable consumption and risk factors for cardiovascular disease. Metabolism: Clinical and Experimental 58 (4): 460-468.

Mullaney, M. I., C. Corish, and A. Loxley. 2009. Exploring the nutrition and lifestyle knowledge, attitudes and behaviors of student home economics teachers: A four year longitudinal study. Journal of Nutrition Education and Behavior 41 (4, Supplement): S8.

Parmenter, K., J. Waller, and J. Wardle. 2000. Demographic variation in nutrition knowledge in England. Health Education Research 15 (2): 163-174.

Parmenter, K., and J. Wardle. 1999. Development of a general nutrition knowledge questionnaire for adults. European Journal of Clinical Nutrition 53 (4): 298-308.

Parmer, S. M., J. Salisbury-Glennon, D. Shannon, and B. Struempler. 2009. School gardens: An experiential learning approach for a nutrition education program to increase fruit and vegetable knowledge, preference, and consumption among second-grade students. Journal of Nutrition Education and Behavior 41 (3): 212-217.

Pirouznia, M. 2001. The association between nutrition knowledge and eating behavior in male and female adolescents in the U.S. International Journal of Food Sciences and Nutrition 52 (2): 127-132.

Polonia, J., and L. Martins. 2009. A comprehensive review on salt and health and current experience of worldwide salt reduction programs. Journal Of Human Hypertension 23 (11): 771-772.

Pratt, C. A., J. Stevens, and S. Daniels. 2008. Childhood obesity prevention and treatment: Recommendations for future research. American Journal of Preventive Medicine 35 (3): 249-252.

Raine, K. D. 2005. Determinants of healthy eating in Canada: An overview and synthesis. Canadian Journal of Public Health 96 (Suppl 3): S8-S14. 
Raykov, T., and G. A. Marcoulides. 2011. Introduction to psychometric theory. New York: Routledge/Taylor \& Francis Group.

Rodrigues, S. S. P., B. Franchini, P. Graça, and M. D. V. de Almeida. 2006. A new food guide for the Portuguese population: Development and technical considerations. Journal of Nutrition Education \& Behavior 38 (3): 189-195.

Ruxton, C. H., E. J. Gardner, and H. M. McNulty. 2010. Is sugar consumption detrimental to health? A review of the evidence 1995-2006. Critical Reviews in Food Science and Nutrition 50 (1): 1-19.

Serra-Majem, L., B. Roman-Vinas, G. Salvador, L. Ribas-Barba, J. Ngo, C. Castell, and C. Cabezas. 2007. Knowledge, opinions and behaviors related to food and nutrition in Catalonia, Spain (1992-2003). Public Health Nutrition 10 (11A): 1396-1405.

Shah, P., A. Misra, N. Gupta, D. K. Hazra, R. Gupta, P. Seth, A. Agarwal, et al. 2010. Improvement in nutrition-related knowledge and behavior of urban Asian Indian school children: Findings from the "Medical Education for Children/Adolescents for Realistic Prevention of Obesity and Diabetes and for Healthy aGeing" (MARG) intervention study. British Journal of Nutrition 104 (3): 427-436.

Sharma, S. V., A. D. Gernand, and R. S. Day. 2008. Nutrition knowledge predicts eating behavior of all food groups except fruits and vegetables among adults in the Paso del Norte region: Que Sabrosa Vida. Journal of Nutrition Education \& Behavior 40 (6): 361-8.

Taylor, J. P., S. Evers, and M. McKenna. 2005. Determinants of healthy eating in children and youth. Canadian Journal of Public Health 96 (Suppl 3): S20-6, S22-9.

Terwee, C. B., S. D. M. Bot, M. R. de Boer, D. A. W. M. van der Windt, D. L. Knol, J. Dekker, L. M. Bouter, and H. C. W. de Vet. 2007. Quality criteria were proposed for measurement properties of health status questionnaires. Journal of Clinical Epidemiology 60 (1): 34-42.

Thorndike, R. M. 1995. Book review: Psychometric theory. 3rd ed. by J. Nunnally and I. Bernstein. Applied Psychological Measurement 19 (3): 303-305.

Viswanath, K., and K. Bond. 2007. Social determinants and nutrition: Reflections on the role of communication. Journal of Nutrition Education E Behavior 39 (2 Suppl): S20-24.

Wardle, J., K. Parmenter, and J. Waller. 2000. Nutrition knowledge and food intake. Appetite 34 (3): 269-275.

WHO. 2009. Interventions on diet and physical activity: What works. Summary report. Rome: World Health Organization.

Worsley, A. 2002. Nutrition knowledge and food consumption: Can nutrition knowledge change food behavior? Asia Pacific Journal of Clinical Nutrition 11 (Suppl 3): $S 579-585$. 